\title{
Les cheminots dans la Résistance. Une exposition
}

Marie-Noëlle Polino

\section{OpenEdition}

\section{Journals}

Édition électronique

URL : https://journals.openedition.org/rhcf/545

DOI : 10.4000/rhcf.545

\section{Éditeur}

Rails \& histoire

Édition imprimée

Date de publication : 1 juin 2006

Pagination : 252-261

ISSN : 0996-9403

\section{Référence électronique}

Marie-Noëlle Polino, "Les cheminots dans la Résistance. Une exposition », Revue d'histoire des chemins de fer [En ligne], 34 | 2006, mis en ligne le 16 mai 2011, consulté le 22 avril 2022. URL : http:// journals.openedition.org/rhcf/545; DOI : https://doi.org/10.4000/rhcf.545

Ce document a été généré automatiquement le 22 avril 2022

Tous droits réservés 


\title{
Les cheminots dans la Résistance. Une exposition
}

\author{
Marie-Noëlle Polino
}

1944-1945, Pierre Perronnet (2e à gauche) avec des collègues du dépôt SNCF de Clermont-Ferrand.

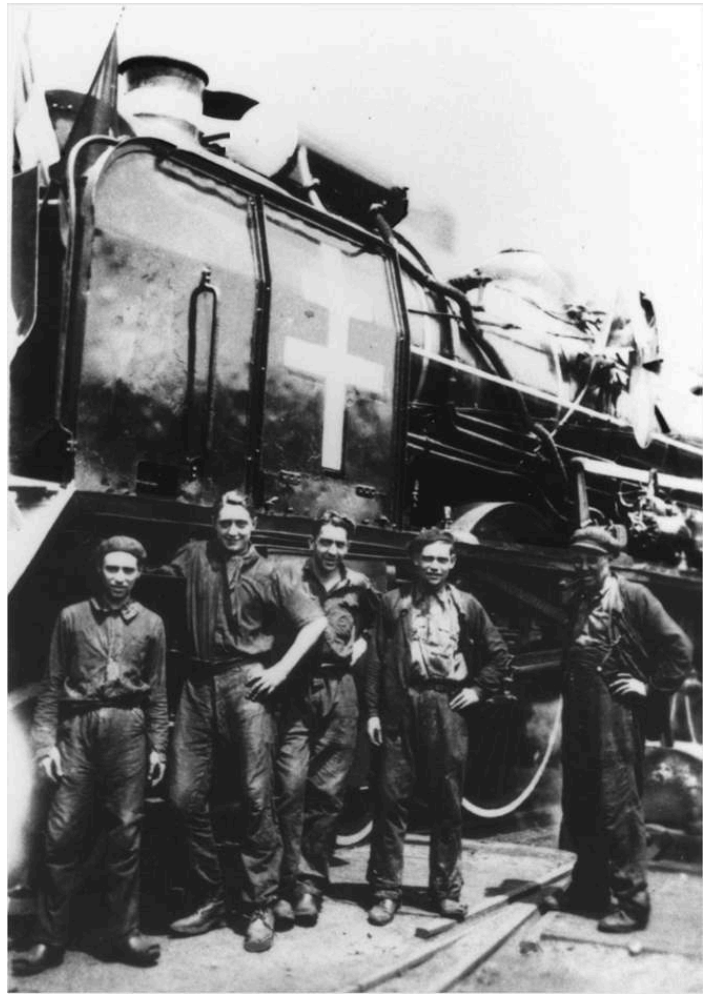

Photographie de l'affiche de l'exposition "Les cheminots dans la Résistance", Paris, Mémorial du Maréchal Leclerc de Hauteclocque et de la Libération de Paris, Musée Jean Moulin, 29 novembre 2005 - 15 avril 2006

Coll. Yves Perronnet. 
1 Le Mémorial Leclerc de Hauteclocque et de la Libération de Paris et le Musée Jean Moulin ont accueilli à Paris, de novembre 2005 à avril 2006, l'exposition Les cheminots dans la Résistance, réalisée par la Fondation de la Résistance, en coopération avec l'AHICF, avec la participation et le soutien de la SNCF. L'exposition est présentée à la Cité du train (Mulhouse) pendant l'été 2006 puis rejoindra les villes, musées de la résistance et de la déportation, établissements d'enseignement, sites ferroviaires qui en feront la demande. Le calendrier de sa circulation sera publié sur le site Internet de l'AHICF.

2 L'exposition Les cheminots dans la Résistance entre dans le cadre d'une convention passée entre la SNCF et la Fondation de la Résistance. Celle-ci, fondation constituée en 1992 et reconnue d'utilité publique par un décret du 5 mars 1993, est placée sous le patronage du Président de la République. Ses deux missions principales sont la sauvegarde de la mémoire de la résistance contre l'occupant entre 1940 et 1945 et la transmission, aux jeunes générations et à la société en général, des valeurs individuelles et collectives qui motivaient les acteurs de la Résistance. Encourager la recherche historique dans ce domaine est un des moyens par lesquelles elle les remplit l'une et l'autre. Par ailleurs, la fondation peut accueillir des personnes physiques ou morales, publiques ou privées, notamment les associations liées à la Résistance désireuses de contribuer à son activité ou de se survivre grâce à elle. C'est ainsi que, lors de sa dissolution en juin 2000, l'association Résistance-Fer, constituée à l'automne 1944, a remis ses collections d'objets et actifs à la fondation tandis qu'elle versait ses papiers aux Archives nationales ${ }^{1}$. À la fin des années 1970, voyant les anciens résistants quitter la SNCF pour la retraite et soucieuse de la transmission du souvenir de l'action résistante aux nouvelles générations de cheminots, l'association avait créé une exposition de panneaux qui a circulé pendant plusieurs années par le courrier interne à la SNCF d'un établissement à l'autre. Ces panneaux se sont retrouvés dans les collections transmises à la fondation de la Résistance et ont fait naître l'idée d'une nouvelle exposition, elle aussi destinée à circuler, mais qui devrait répondre aux critères actuels de ce type de support avec, surtout, un contenu renouvelé grâce aux fonds d'archives aujourd'hui accessibles et aux recherches historiques les plus récentes.

3 L'exposition s'inscrit dans le mouvement général de transmission de la mémoire des témoins, peu à peu disparus, aux historiens qui ont à présent de la Résistance une vision d'ensemble - le Dictionnaire de la Résistance paru en avril 2006 en fait foi ${ }^{2}$ - en ce qu'elle est l'œuvre d'un comité d'historiens qui ont eu pour projet d'établir un bilan de nos connaissances sur les cheminots dans la Résistance ${ }^{3}$. Le titre choisi disait déjà la volonté de questionner l'image d'une « résistance des cheminots » et de restituer la multiplicité des cas individuels et des démarches personnelles, des organisations aussi qui ont fait appel aux cheminots pour le motif de leur profession et de ce que celle-ci impliquait pour l'action résistante.

4 L'exposé de ce bilan devait laisser voir le travail de recherche dont il était issu et faire état aussi bien des faits avérés que des sujets pouvant prêter aujourd'hui à des interprétations diverses voire opposées. En insistant sur la présentation de documents inédits ou trop dispersés pour avoir jamais été publiés ensemble, il lance un appel aux témoins et aux détenteurs d'archives de la résistance, de la déportation et plus largement de la Deuxième Guerre mondiale pour les inciter à les sauvegarder en les faisant connaître et en les confiant aux archives publiques ${ }^{4}$. Enfin, la forme adoptée pour l'exposition, 50 panneaux riches d'un texte important en volume et reproduisant 
les documents originaux, porte la volonté de sa circulation la plus large possible pour qu'elle soit connue de publics variés autant par la géographie que par l'âge ou la profession, en privilégiant d'une part les jeunes, lycéens et étudiants principalement, d'autre part les cheminots actifs et retraités ${ }^{5}$.

5 Ce projet se traduit par deux caractères particuliers à l'exposition: son étendue, d'abord. Enchaînant sept chapitres - la SNCF dans la France en guerre ; s'engager ; des formes d'action multiples ; les cheminots et la résistance organisée ; les cheminots dans la libération; les cheminots face à la répression; la mémoire -, l'exposition dépasse de beaucoup le cadre de l'occupation allemande.

6 L'importance du contexte s'est justifiée par la demande du public. Il comprend la création, par un décret du 31 août 1937 prenant effet au $1^{\mathrm{er}}$ janvier 1938, de la SNCF ; la mobilisation de l'entreprise et des cheminots, leur rôle précisément dans la mobilisation générale, dans l'exode; la communauté professionnelle en 1938 et ses caractères propres. Si l'histoire de la Deuxième Guerre mondiale doit être rappelée dans un souci pédagogique, il en va de même du système technique ferroviaire et des conditions de travail de l'entre-deux-guerres qui ont été oubliés et ne font plus partie du savoir partagé du public : c'est pourquoi la projection du documentaire Ceux du rail, tourné en 1942, qui raconte la journée de travail d'une équipe de conduite, mécanicien et chauffeur, a été incluse dans la présentation parisienne de l'exposition ${ }^{6}$. Il s'agit aussi et surtout de faire ressortir la résistance et sa définition progressive de ce tableau général : ni «travail au ralenti »; ni " geste occasionnel », même si s'ils sont passibles d'une répression aussi forte que d'autres, la résistance est l'action qui résulte d'un engagement. Celui-ci ne se résume pas à l'affiliation à un mouvement ou à un réseau : il peut exister des résistants isolés et qui le resteront, d'autres travaillent pour plusieurs organisations. Le parti est donc pris de restituer des itinéraires singuliers qui vont démontrer à chaque étape du discours la diversité de ces engagements et des formes d'action qui se succèdent pendant quatre ans. Enfin, la guerre, dans le contexte de laquelle se trouve la SNCF, ne doit jamais être oubliée avec ce qu'elle impose de contraintes : un territoire émietté par les lignes séparant les différentes zones - lignes que le cheminot peut franchir; une pénurie omniprésente qui envahit la vie quotidienne - le cheminot peut circuler entre villes et entre villes et campagnes et transporter du ravitaillement; une absence complète d'information que nous ne parvenons que difficilement à nous représenter - le cheminot peut seul connaître certains faits et transmettre presse, courrier et messages clandestins.

7 À l'autre extrémité de l'exposition, l'importance donnée à la mémoire de la Résistance a pu également surprendre. Outre l'influence de l'historiographie actuelle, qui inclut dans l'histoire d'un événement ses représentations et sa transmission, il faut y voir un trait particulier non seulement à la résistance, mais à son histoire dans le monde cheminot. L'importance du rôle de l'association Résistance-Fer et de son identification réussie à la résistance organisée des cheminots dans la SNCF, puis la critique de cette construction par d'autres acteurs de la Résistance (notamment les organisations de la mouvance communiste), ont conduit les auteurs de l'exposition à retracer précisément l'évolution du souvenir de la Résistance et son utilisation par les forces politiques de l'après-guerre - un fait historique et une forme d'action justifiés par leur contexte comme ceux qui les ont précédés, les motivations des acteurs d'alors n'étant pas forcément synonymes de volonté de «récupération» condamnable sans examen. 
Surtout, il s'agit d'appeler le public, y compris les professionnels de l'histoire, à revenir sur sa représentation des cheminots dans la résistance.

8 La volonté de montrer les lacunes de la connaissance et les ombres qui entourent certaines questions historiques est le deuxième trait particulier à cette exposition.

Venue soixante ans après la libération et plusieurs centaines, sinon milliers de titres parus sur la Résistance, l'exposition intègre et diffuse des connaissances qui ne pouvaient être celles des rédacteurs des années 1970. Ainsi, elle explore l'application aux cheminots du concept de "fonctionnalité », défini par François $\operatorname{Marcot}^{7}$, à la lumière de ce qu'on sait à présent d'autres corporations, Georges Ribeill en donne un exemple dans le présent volume. Cependant, une étude quantitative exhaustive, une statistique de la présence des cheminots dans la résistance sont et resteront impossibles, par manque de sources, mais surtout de l'accord de celles-ci sur la définition de l'action résistante. Certes, faute de mieux, l'exposition présente les chiffres résultant des quelques monographies départementales existantes, fondées sur la prise en compte des cartes des «Combattants volontaires de la Résistance ». Mais ces sources postérieures à la guerre, qui correspondent aux demandes de reconnaissance de services rendus ouvrant droit à pension, sous-estiment dans une mesure qui reste à apprécier la résistance de type non militaire. Plutôt que d'écrire une histoire qui partirait de la SNCF pour établir et qualifier la résistance de ses agents, une démarche faite en son temps par Paul Durand ou avant lui par les scénaristes de Bataille du rail qui a pour résultat la prédominance de l'image d'une «entreprise résistante ", les auteurs de l'exposition, spécialistes de la résistance mais non des cheminots, même s'ils le sont devenus ${ }^{8}$, sont partis de tout ce que nous savons aujourd'hui de la Résistance pour trouver des traces avérées des engagements individuels des cheminots dans les différentes organisations clandestines. Elles permettent de dégager la spécificité d'une corporation sollicitée pour à peu près toutes les formes d'action résistante, ce qui explique l'implication progressive de cheminots de tous métiers, de tous grades, de tous âges. Elles montrent aussi l'importance de la diversité des itinéraires individuels avant et pendant la période de la Résistance, au-delà de l'appartenance commune à l'entreprise.

Inversement, l'exposition met en valeur des faits nouveaux, soit qu'ils aient été connus ou reconnus tardivement, soit que la synthèse de faits dispersés permette à présent d'insister davantage sur des traits de l'action résistante des cheminots qui ne tenaient pas jusqu'ici le devant de la scène historique: il en est ainsi du rôle essentiel des cheminots dans l'information et la diffusion de la presse clandestine, acheminée par le système de l'enregistrement des bagages, alors général; du rôle des syndicats clandestins et « légaux » et des rapports entre les deux types d'organisations, souvent animées par les mêmes militants; de l'application à et par la SNCF des lois d'exclusion de Vichy, en particulier antisémites, et de la répression anti-communiste depuis 1939; de l'histoire des sabotages de voies, enfin, trop souvent, à tort, assimilés à l'action des cheminots dont ils seraient la forme exclusive. S'ils ont renseigné les unités de saboteurs tant sur les cibles que sur les moyens (sabotage par détirefonnage), les cheminots eux-mêmes ont rarement été impliqués dans ces actions avant l'application du plan vert à l'été 1944. Quant à l'utilisation du plastic parachuté par les alliés, elle est tardive. En revanche, la mise hors service du matériel roulant dans les ateliers, par des moyens qui en retardent l'effet, est bien une action cheminote, avec l'exception de 
l'action directe comme celle qu'a entreprise avec succès le groupe auquel appartient Georges Delepaut à Tourcoing.

11 La possibilité des déportations, ou du moins le fait que rien n'a été opposé à la circulation des trains, en particulier ceux, les derniers, qui sont encore partis vers l'Allemagne à l'été 1944, fait partie des interrogations qui s'adressent à toute la période et à tous ses acteurs mais qui, comme le montrent les analyses de Christian Chevandier, ont été surtout dirigées en France vers les cheminots comme groupe professionnel. L'exposition, dans sa première partie, inclut les trains de la déportation dans les contraintes imposées à la SNCF par l'occupant; au titre des formes d'action, elle mentionne l'aide ponctuelle apportée aux personnes déportées pendant leur trajet en France - transmission de messages, par exemple ; enfin, au chapitre de la libération, elle décrit les derniers trains qui sont parvenus jusqu'en Allemagne, comme celui parti de Toulouse au début de juillet 1944, et ceux qui ont été arrêtés, soit sur ordre (Péronne), soit par un détournement suivi d'un assaut des forces locales de résistance aidées par les alliés (Annonay), en rappelant le contexte militaire de l'époque qui n'était pas favorable à la généralisation de telles actions. L'exposition n'oppose pas résistance et déportation -car elles ne s'annulent pas, ni dans un sens, ni dans l'autre. Elle n'ignore ni ne nie, mais est bien loin aussi de la dénégation, comme une interprétation en termes uniquement militaires de la résistance aurait pu y être encline dans l'après-guerre, les victimes civiles étant considérées alors comme les dommages collatéraux de l'action militaire. Elle rappelle la complexité de la position des cheminots qu'ils doivent à leur appartenance professionnelle, celle-là même qui leur a donné des moyens d'action'.

Pour toutes ces raisons, une telle exposition était nécessaire et la forme qu'elle a adoptée doit lui garantir la publicité qu'elle mérite ${ }^{10}$. Son caractère pédagogique - elle a été plébiscitée par les enseignants qui l'ont visitée -, son efficacité dans la diffusion de la recherche historique sont avérés, ainsi que la force des témoignages transmis et des documents choisis. Alliant mémoire et histoire, institutions dédiées à l'une et à l'autre et volonté d'une entreprise, on peut dire qu'elle réussit son pari d'actualité tout en étant un hommage au passé qu'elle contribue à faire plus exactement connaître. 
Figure 1.Une vue de l'exposition dans la présentation parisienne.

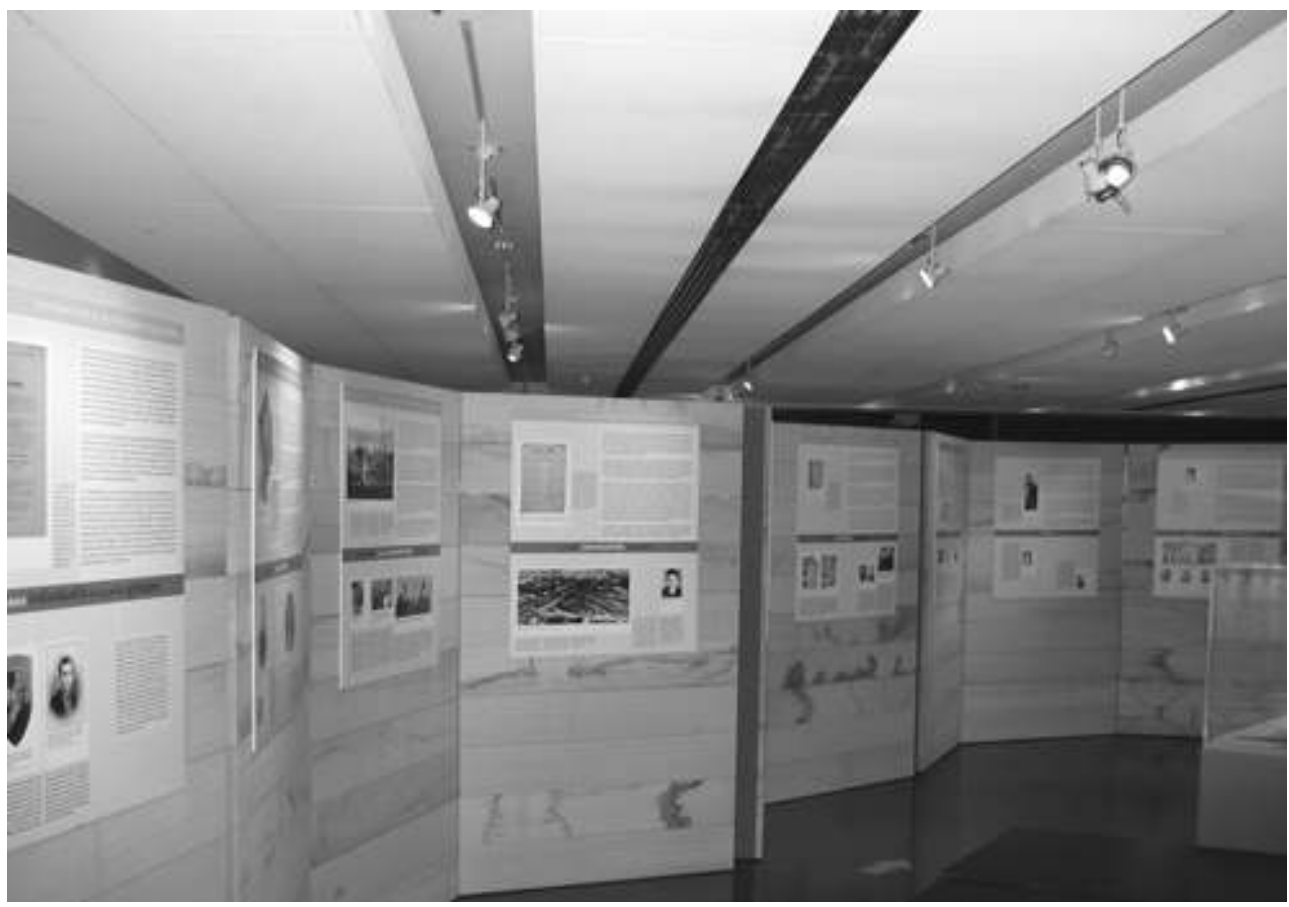

La Fondation de la Résistance avait prêté des objets de la collection Résistance-Fer (faux papiers, brassards, diplômes et citations d'après guerre) et quelques panneaux de l'exposition réalisée par cette association en 1978-1980. Chaque institution qui recevra l'exposition est invitée à l'enrichir par des éléments se rapportant à l'action des cheminots locaux dans la Résistance.

Cl. AHICF / M.-N. Polino. 
Figure 2

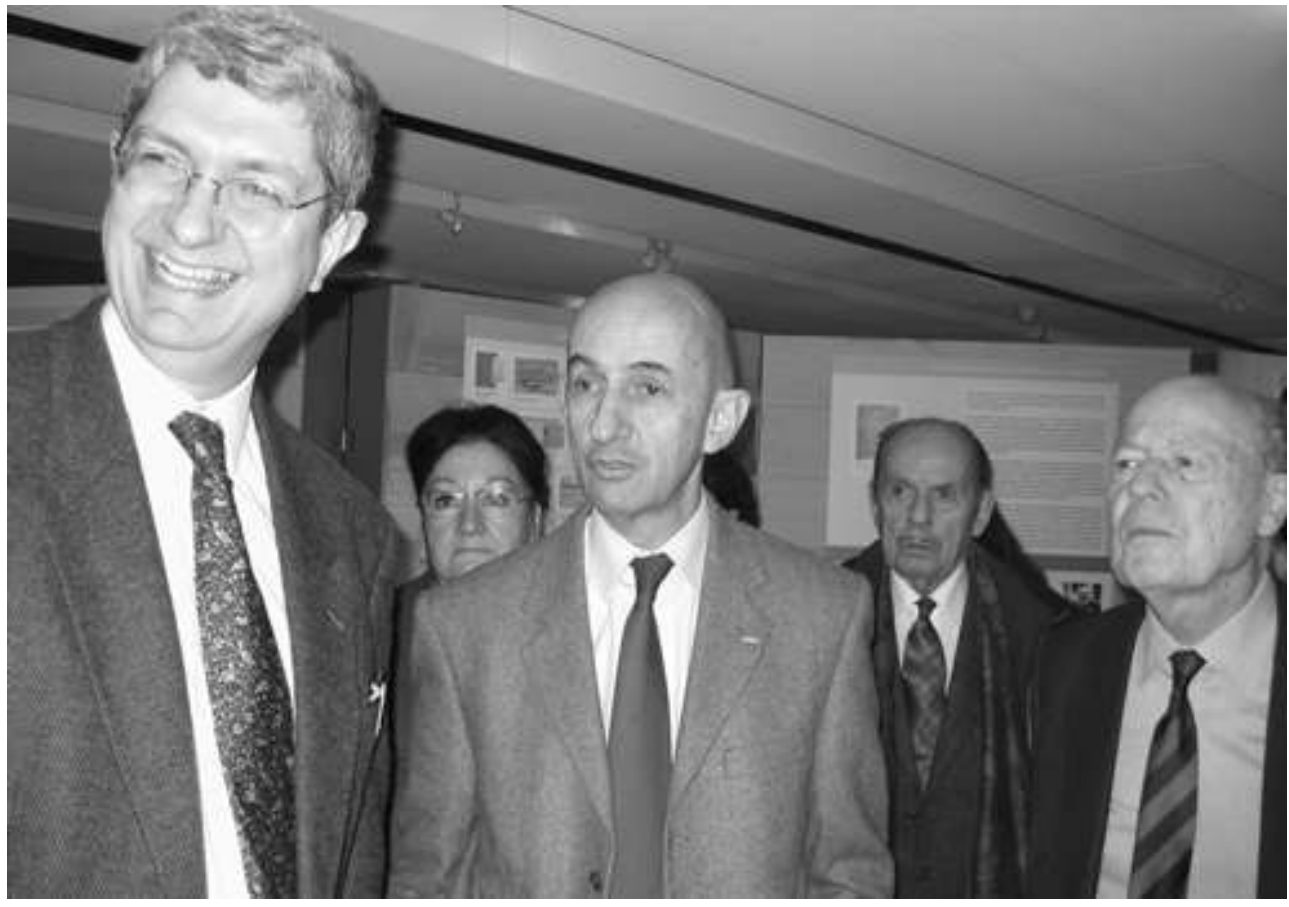

Odette Christienne, adjointe au maire de Paris, chargée de la mémoire, du monde combattant et des archives, Louis Gallois, président de la SNCF, Pierre Morel, président du Comité d'action de la Résistance et Pierre Sudreau, ancien ministre, vice-présidents de la Fondation de la Résistance, visitent l'exposition le jour de son inauguration parisienne sous la conduite de Bruno Leroux (à gauche).

Cl. AHICF / M.-N. Polino

Figure 3. Le panneau "1939-1940. Une mobilisation totale face à la guerre" et le panneau "Les derniers trains de la déportation" de l'exposition Les cheminots dans la Résistance.
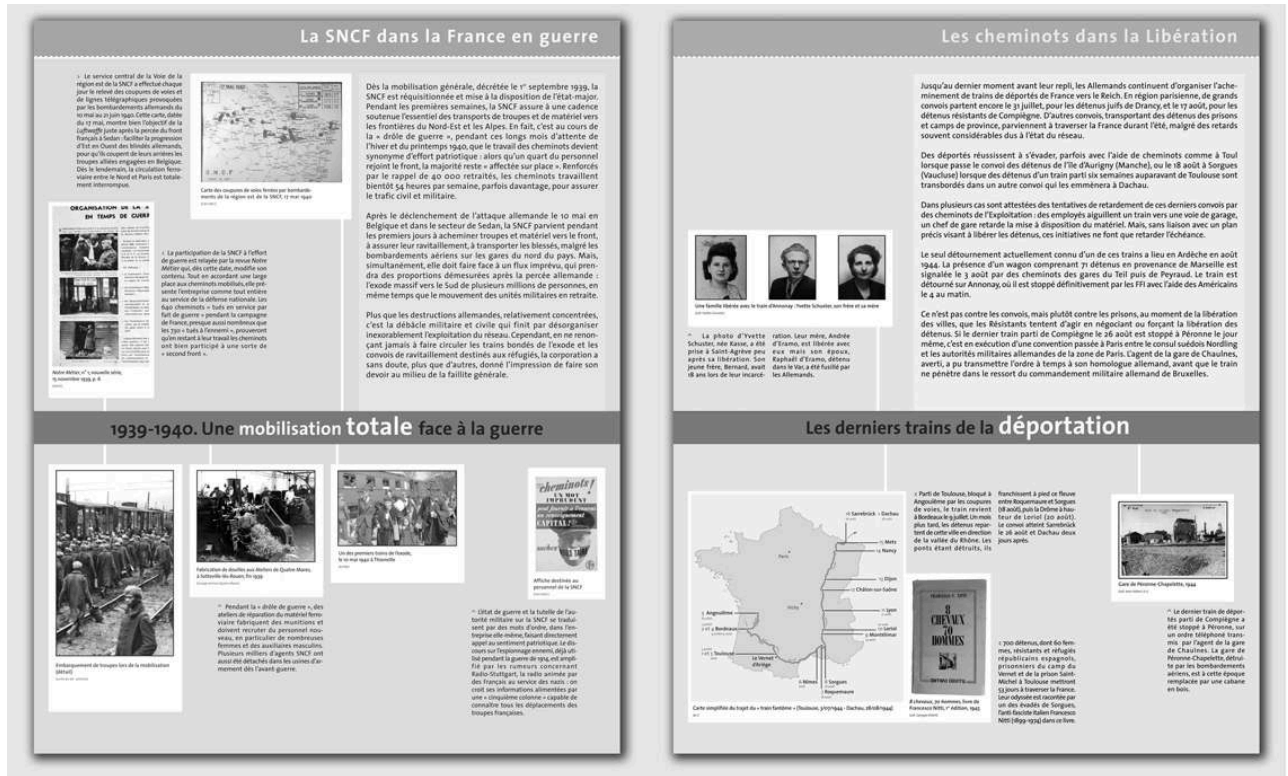

Le graphisme des panneaux engage le lecteur à différencier les chapitres et à suivre le déroulement chronologique du discours. II privilégie certains documents particulièrement utiles ou frappants : cartes, photographies, affiches.

(c) Fondation de la Résistance 


\section{NOTES}

1. Voir l'inventaire de ce fonds en ligne sur le site www.ahicf.com.

2. François Marcot (dir.), avec la collab. de Bruno Leroux et de Christine Levisse-Touzé, Dictionnaire historique de la Résistance, Paris, Robert Laffont, coll. «Bouquins », 2006, 1248 p.

3. Les textes sont dus à Bruno Leroux, directeur historique de la Fondation de la Résistance, et à Cécile Hochard, docteur en histoire, chargée de mission à la fondation pendant l'année 2003-2004. Ils étaient entourés d'un comité de pilotage réunissant représentants de la SNCF, de la fondation, madame Christine Lévisse-Touzé, directeur du Mémorial Leclerc de Hauteclocque et de la Libération de Paris et du Musée Jean Moulin et Marie-Noëlle Polino pour l'AHICF. Le comité historique de l'exposition était constitué de: Christian Chevandier, maître de conférences à l'université de Paris I - Panthéon-Sorbonne; Laurent Douzou, professeur d'histoire contemporaine, Institut d'études politiques de Lyon; Georges Ribeill, directeur de recherche à l'ENPC ; Serge Wolikow, professeur d'histoire contemporaine à l'université de Bourgogne.

4. Une campagne nationale de sensibilisation des détenteurs d'archives privées de la résistance et de la déportation (particuliers, responsables d'associations, de musées associatifs) a été lancée par la Fondation de la Résistance, la Fondation pour la mémoire de la Déportation, les ministères de la Culture et de la Défense pour les inciter à déposer leurs fonds dans les archives publiques afin d'assurer leur pérennité et leur ouverture à la recherche. Contact: Frantz Malassis, responsable Archives, Fondation de la Résistance, 30, boulevard des Invalides, 75007 Paris, 014705 6787, frantzmalassis@club-internet.fr.

5. L'exposition est accompagnée d'un catalogue qui en reproduit la plupart des textes et documents dans un format qui en rend la lecture aisée et à un prix voulu modique : "Les cheminots dans la Résistance ", La Lettre de la Fondation de la Résistance, numéro spécial, 2005, 32 pages, 4,50 euros.

6. Les auteurs de l'exposition ont fait figurer en fin de catalogue un glossaire des termes appartenant à l'histoire de la Deuxième Guerre mondiale et à l'histoire et à la technique ferroviaires. Cependant, la signification du mot «tender », qui n'y figure pas, a été la question la plus couramment posée aux conférenciers lors de l'explication des moyens par lesquels les clandestins passaient les lignes de démarcation.

7. Voir François Marcot, «Pour une sociologie de la Résistance: intentionnalité et fonctionnalité ", in Antoine Prost (dir.), La Résistance, une histoire sociale, Paris, Les Éditions de l'atelier/Les Éditions ouvrières, 1997, p. 21-41 et Christian Chevandier, «La résistance des cheminots : le primat de la fonctionnalité plus qu'une réelle spécificité », ibid., p. 147-158.

8. Comme le montre la bibliographie établie par Cécile Hochard, co-auteur de l'exposition, dans le présent volume (en ligne sur le site : www.ahicf.com)

9. L'itinéraire de Bernard Le Chatelier, qui est rappelé au chapitre "répression ", en est un exemple éclairant : chef du dépôt qui fournit les machines et les équipes de conduite pour les trains qui emmènent vers l'Allemagne depuis Compiègne les détenus du camp de Royallieu, résistant appartenant à deux réseaux de renseignement sans quitter son poste, il part lui-même en déportation dans un de ces trains. Voir son ouvrage, Matricule 51306, mémoires de déportation, récit, Paris, Les Éditions de la Bruyère, 1984, 192 p. (2 éd., 2005), dont nous avions reproduit sous le titre « les paradoxes de la résistance professionnelle » un extrait, commenté par un entretien avec l'auteur, dans le recueil «Les cheminots dans la guerre et l'occupation. Témoignages et récits ", Revue d'histoire des chemins de fer hors série, $\mathrm{n}^{\circ} 7,2^{\mathrm{e}}$ éd. revue et augmentée, 2004, p. $149-159$.

10. L'itinérance de l'exposition est régie par la Fondation de la Résistance, à laquelle il convient de s'adresser pour en demander le prêt (30, boulevard des Invalides, 75007 Paris). 


\section{AUTEUR}

MARIE-NOËLLE POLINO

Secrétaire scientifique de l'AHICF 